\title{
Histological, immunohistochemical and ultrastructural study of secondary compressed spinal cord injury in a rat model
}

\author{
Wafaa S. Ramadan ${ }^{1,2,3}$, Ghada A. Abdel-Hamid ${ }^{1,2,4}$, Saleh Al-Karim ${ }^{2,5}$, Aymn T. Abbas ${ }^{6,7}$ \\ ${ }^{1}$ Department of Anatomy, Faculty of Medicine, King Abdulaziz University, Jeddah, Saudi Arabia \\ ${ }^{2}$ Embryonic Stem Cell Unit, King Fahd Medical Research Center, King Abdulaziz University, Jeddah, \\ Saudi Arabia \\ ${ }^{3}$ Department of Anatomy, Faculty of Medicine, Ain Shams University, Cairo, Egypt \\ ${ }^{4}$ Department of Anatomy, Faculty of Medicine, Suez Canal University, Ismaillia, Egypt \\ ${ }^{5}$ Department of Biological Sciences, Faculty of Science, King Abdulaziz University, Jeddah, Saudi Arabia \\ ${ }^{6}$ Special Infectious Agent Unit, KFMRC, King Abdulaziz University, Jeddah, Saudi Arabia \\ ${ }^{7}$ Biotechnology Research Laboratories, Gastroenterology Surgery Center, Mansoura University, \\ Mansoura, Egypt
}

\begin{abstract}
Introduction. Spinal cord injury (SCI) is a life-disrupting condition in which the first few days are the most critical. Secondary conditions remain the main causes of death for people with SCI. The response of different cell types to SCI and their role at different times in the progression of secondary degeneration are not well understood. The aim of this study was to study the histopathological changes of compressed spinal cord injury (CSCI) in a rat model.

Material and methods. Forty adult male Sprague-Dawley rats were divided into four groups. In group I, the rats were left without any surgical intervention (control). In group II, the rats were subjected to laminectomy without spinal cord compression (sham-operated). In group III, the rats were sacrificed one day after CSCI. In group IV, the rats were sacrificed seven days after CSCI. The light microscopy was employed to study the morphology using H\&E, osmic acid staining and immunohistochemistry to detect glial fibrillary acidic protein (GFAP). The electron microscopy was applied for ultrastructure study.

Results. Histopathological examination of the posterior funiculus of the white matter revealed minute hemorrhages and localized necrotic areas on day 1, which transformed to areas of cavitation and fibrinoid necrosis surrounded by a demarcating rim of numerous astrocytes by day 7. The mean percentage of area of GFAP expression increased significantly by day 7. Osmic acid staining revealed swollen nerve fibers after one day, while numerous fibers had been lost by day 7. An ultrastructure study revealed swollen redundant thinned myelin and myelin splitting, as well as degeneration of axoplasm on day 1 . On day 7, layers of the myelin sheath were folded and wrinkled with partial or complete demyelination areas. The myelin lamellae were disorganized and loose. The G-ratio was significantly greater on day 1 than day 7 after CSCI.

Conclusions. In the rat model of CSCI details of the progressive spinal cord injury can be analyzed by morphological methods and may be helpful in the identification of the onset and type of clinical intervention.

(Folia Histochemica et Cytobiologica 2017, Vol. 55, No. 1, 11-20)
\end{abstract}

Key words: rat; compressed spinal cord injury; morphology; GFAP; myelin fibers; electron microscopy

Correspondence address: Prof. W.S. Ramadan

Department of Anatomy Faculty of Medicine

King Abdulaziz University

P.O. Box: 42806, Jeddah 21551 KSA, Saudi Arabia

e-mail:wramadhan@kau.edu.sa 


\section{Introduction}

Spinal cord injury (SCI) refers to damage to the spinal cord, traumatic or non-traumatic, resulting in severe dependency of affected persons and economic burden both for individuals and society. In low-income countries, preventable secondary conditions remain the main cause of death for people with SCI [1].

The common mechanisms of injury in human spinal cord (SCI) are compression, contusion, laceration, and transection. No single animal model can imitate all of these various mechanisms [2]. Although transection models provide an idealized setting for studying spinal cord regeneration across a complete lesion, transected spinal cords are rarely encountered in human SCI. In other words, most injured spinal cords maintain some tissue continuity across the area of injury. Accordingly, contusion and compression models are more clinically relevant [3].

It is well known that low regeneration in the central nervous system is a major problem in recovery especially from traumatic injuries. Several mechanisms have been identified following trauma. The secondary phase of SCI starts minutes after the injury and lasts for weeks. It involves both the continuation of some events that began in the acute phase and some new events [4]. It is the first few days after injury that are the most crucial in terms of survival, neuroprotection, prevention of secondary complications, and psychosocial adjustment to the drastic change in life circumstances [5]. Although certain probable initiating factors of secondary degeneration have been identified, the response of different cell types to SCI in the progression of secondary degeneration has not been well understood [6]. Demyelination is one of the major pathological changes manifested in compressed spinal cord injury (CSCI) [7]. It results in disrupted motor coordination, spasticity, and incontinence [8]. Studies of the pathological mechanisms of demyelination related to CSCI have been limited in the past by the deficient in vivo models.

The aim of this study was to define the histopathological changes in the course of CSCI with special attention on the ultrastructural alterations for a better comprehension of the pathological consequences to help clinicians determine the onset and the plan of the presumed treatment.

\section{Material and methods}

Animals. A total of 40 adult male Sprague-Dawley rats weighing $200 \mathrm{~g}$ to $250 \mathrm{~g}$ were provided by the animal house of King Fahd Medical Research Center (KFMRC), King Abdulaziz University, Jeddah, Saudi Arabia. All procedures were conducted in accordance with guidelines and protocols reviewed and approved by the ethical committee for animal care and use at KFMRC, which are in accordance with the guidelines of the Canadian Council on Animal Care. The rats were housed at $22-24^{\circ} \mathrm{C}$ and $55 \%$ relative humidity, with a 12:12 light:dark photoperiod. The rats were fed a commercial diet and given water ad libitum. All rats were anesthetized by intraperitoneal injection of sodium pentobarbital ( $50 \mathrm{mg} / \mathrm{kg}$ body weight, Sigma-Aldrich, St. Louis, MO, USA).

Experimental design and surgical procedure. The animals were divided randomly into four groups of ten rats each. Group I (control): rats were left without surgical intervention in order to study the normal structure of the spinal cord in rats at the same level as the SCI. Group II (sham-operated): animals were subjected to the laminectomy only, without spinal cord compression. Group III: rats were sacrificed one day after CSCI. Group IV rats were sacrificed seven days after CSCI.

The spinal cord contusion was performed using the clip compression technique as described by Bakar et al. [9]. All animals were sedated with intramuscular injection of $40 \mathrm{mg} / \mathrm{kg}$ ketamine and $5 \mathrm{mg} / \mathrm{kg}$ xylazine on spontaneous respiration at room temperature. A dorsal laminectomy at level 9-10 of the thoracic vertebra was performed on all animals. The spinal cord was exposed, and except for the sham group, SCI was induced using a temporary aneurysm clip (Mizuho Aneurysm Clip, Mizuho, Japan) for $60 \mathrm{sec}-$ onds. The incision was then gradually sutured in layers. The rats were allowed to recover from the anesthesia and housed. Antibiotics (enrofloxacin, $2.5 \mathrm{mg} / \mathrm{kg}$, s.c.) were given before surgery and daily for 3-5 days. Saline (2-5 mL i.p.) and nutritional supplements (Nutri-Cal, EVSCO Pharmaceuticals, Buena, NJ, USA) were given for 3-5 days after CSCI.

\section{Morphological evaluation of CSCI}

Sampling of tissues. One day and seven days after CSCI, the rats were anesthetized and sacrificed. The spinal cord tissues were removed rapidly and sliced coronally at $2 \mathrm{~mm}$ intervals. Spinal cord sections from T9-10 $(1 \mathrm{~cm})$ were divided into four segments. The first segment was immersed in 10\% neutral buffered formalin. Specimens were processed and embedded in paraffin wax.

Light microscopy studies. The $4 \mu \mathrm{m}$-thick sections were stained with hematoxylin and eosin (H\&E) [10], examined using Olympus light microscope BX51TF (Olympus, Tokyo, Japan), and photographed.

Immunohistochemistry of glial fibrillary acidic protein (GFAP). The tissue sections were deparaffinized and dipped in phosphate-buffered saline (PBS, pH 7.4) (Sigma-Aldrich), kept in cold methanol (BDH Laboratory Supplies, Poole, UK) at $2^{\circ} \mathrm{C}$ for $4 \mathrm{~min}$, and washed in PBS several times. The tissues were treated for 30 min with $3 \% \mathrm{H}_{2} \mathrm{O}_{2}$ (Fluka Chemika, Buchs, Switzerland) and then washed with PBS before 
the primary antibody, rabbit anti-GFAP diluted 1:20 in PBS (Sigma-Aldrich) was added, and incubated for $1 \mathrm{~h}$. For control, the primary antibody was omitted before adding the secondary antibody, biotin anti-rabbit, which was diluted 1:20 in PBS containing 1\% bovine serum albumin (BSA; Sigma-Aldrich) for $30 \mathrm{~min}$. Slides were then washed in PBS before adding the extra avidin-peroxidase (Sigma-Aldrich) diluted 1:20 in PBS containing 1\% BSA for $30 \mathrm{~min}$. The slides were then washed in PBS and treated with diaminobenzidine (Bio-Rad Laboratories, Toronto, Canada) for $30 \mathrm{sec}$. Finally, the slides were washed in distilled water, dried, placed in xylene for $5 \mathrm{~min}$ and mounted with DPX (Fluka Chemika) [11].

Quantitative analysis of glial fibrillary acidic protein expression. The slides were digitized using an Olympus digital camera installed on an Olympus BX51TF microscope with a $\times 1 / 2$ photo adaptor using a $\times 40$ objective. The resulting images were analyzed on an Intel Core I3-based computer using Video Test Morphology software (Video Test, St. Petersburg, Russia) with a specific built-in program for immunohistostaining analysis. The system measured the percentage area of GFAP-positive expression. The software routine for quantification was as follows. Step 1: Live images were transferred from the camera to the computer using a U-Tech frame grabber. The required images were captured as a snapshot and saved in $1024 \times 768$ dpi TIFF format. Step 2: Automatic image adjustment (including automatic color balance and contrast) was performed to obtain a high-contrast image and a well-defined range of the target stain. This also reduces background interference with the target stain, which could otherwise affect the result. Step 3: The enhanced image with high image detail was then subjected to automatic thresholding using the target stained area to adjust the threshold level. This converts the target stained area to a binary red mask that overlays the original image. This area is called the region of interest (ROI). The system uses this red mask to calculate the area percentage of the target stain [12].

Osmic acid staining. The third segment was used for osmic acid staining. The spinal cord sections $(1 \mathrm{~cm})$ were fixed with $4 \%$ paraformaldehyde for $24-48 \mathrm{~h}\left(\right.$ at $\left.4^{\circ} \mathrm{C}\right)$ and dehydrated with $30 \%$ sucrose overnight $\left(4^{\circ} \mathrm{C}\right)$. Afterwards the specimens were removed from $4 \%$ paraformaldehyde and each was rinsed with $0.01 \mathrm{~mol} / \mathrm{L}$ phosphate-buffered saline (PBS, pH 7.4) for $20 \mathrm{~min}$. They were then transferred to $1 \%$ osmic acid for 3 to 7 days, and then rinsed with $0.01 \mathrm{~mol} / \mathrm{L}$ PBS (pH 7.4) and stored in $75 \%$ ethanol. This was followed by gradual alcohol dehydration, and embedding in paraffin. Blocks were cut into sections using a conventional microtome (Leica CM1900, Leica Camera AG, Oskar-Barnack, Germany) [13].

Electron microscopy. The fourth segment from the CSCI site was immersed in $4 \%$ paraformaldehyde followed by immediate fixation in 3\% glutaraldehyde and $1 \%$ paraform-

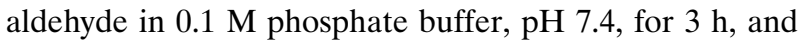
then postfixed in $1 \%$ osmium tetroxide in the same buffer for $2 \mathrm{~h}$. The samples were dehydrated and embedded in epoxy resin mixture. Semithin sections of $1 \mu \mathrm{m}$ thickness were stained with toluidine blue [14]. Ultrathin sections of $70 \mathrm{~nm}$ thick were cut using a diamond knife. The sections were stained with uranyl acetate followed by lead citrate and examined at $80 \mathrm{kV}$ with a JEM-2000EX transmission electron microscope (JEOL, Tokyo, Japan) at King Fahd Medical Research Center.

Determination of G-ratios. All specimens were examined under a transmission electron microscope (JEM-2000EX; JEOL, Tokyo, Japan) and the images were acquired through a SIA-8 CCD camera mounted on the microscope for examination with Northern Eclipse Software (Cheektowaga, NY, USA). For morphological analysis, the images thus acquired were analyzed so as to calculate the G-ratio as the ratio of axon diameter to total fiber diameter [15].

Statistical analysis. The data were statistically analyzed using SPSS statistical software, version 19.0 (SPSS Inc., Chicago, IL, USA) for Windows. The results were presented as means \pm SDs. The differences between the continuous data were analyzed using one-way ANOVA. When equal variance could be assumed, the LSD $t$-test was applied. Data was presented as means \pm standard deviations (SD). $\mathrm{P}<0.05$ was considered significant.

\section{Results}

\section{Effects of CSCI on the morphology of spinal cord 1 and 7 days after CSCI at the light microscopy level} In H\&E-stained sections, the posterior funiculus in the white matter of the spinal cord of the control and sham groups revealed a normal structure, except for a few dilated capillaries in sections of sham group (Fig. 1A, B). One day after the CSCI, minute hemorrhages scattered throughout the punched out areas were noted. Scattered astrocytes and myelin loss in axonal tracts were also observed (Fig. 1C, D). Reactive astrocytosis and diffuse degeneration were evident seven days after the CSCI. Moreover some sections revealed extended areas of fibrinoid necrosis and broadened areas of cavitation (Fig. 1E, F).

In sections immunostained for GFAP, the posterior funiculus of the white matter of the spinal cord of rats from the control and sham groups showed dispersed astrocytes (Fig. 2A, B). On day 1 after the $\mathrm{CSCI}$, the astrocytes appeared reactive with thick cytoskeletal processes on the lesion level (Fig. 2C). Seven days after CSCI circumscribed areas of reactive astrocytosis and numerous astrocytes with thick cytoskeletal processes were observed (Fig. 2D). The 

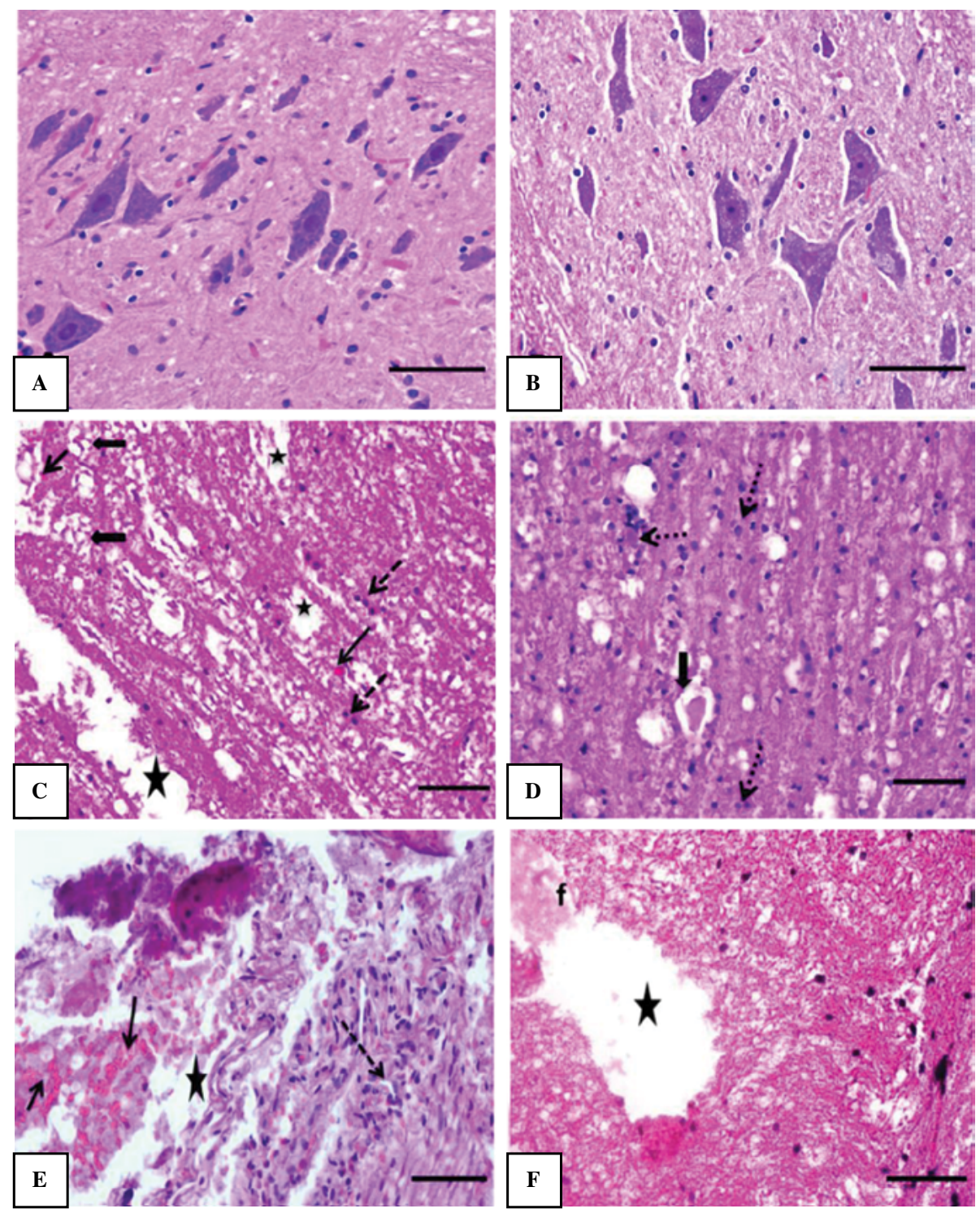

Figure 1. Sections of white matter of the spinal cord of control rats and animals subjected to compressed spinal cord injury (CSCI) as seen by hematoxylin and eosin (H\&E) staining. Normal structure in control (A) and sham-operated (B) rats. C and D: one day after CSCI. In (C) minute hemorrhages (thin arrows) scattered among punched out areas (star) and diffuse degeneration (thick arrows) are present. In (D) increased dispersed astrocytes (dashed arrows) and myelin loss in axonal tracts (thick arrow) are visible. E and F: seven days after CSCI. In (E) extended areas of hemorrhages (thin arrows), areas of cavitation (star) surrounded by reactive astrocytosis (dashed arrows) are present. In (F) extended areas of fibrinoid necrosis and large cavitation areas (star) are visible. Scale bars A and B, $61 \mu \mathrm{m} ; \mathrm{C}-\mathrm{F}, 50 \mu \mathrm{m}$.

expression of GFAP in day 7 CSCI groups was up-regulated as evidenced by a significant increase in the mean percentage area of GFAP expression (Fig. 2E).

In sections stained with osmic acid, the T9-10 spinal cords from the control and sham groups showed darkly stained intact myelin sheaths reflecting the normal myelination of nerve fibers (Fig. 3A, B). Nerve fibers became swollen, and the spaces between fibers apparently decreased one day after CSCI (Fig. 3C). At seven days after compression, numerous fibers were lost (Fig. 3D).

\section{Effects of CSCI on the morphology of spinal cord 1 and 7 days after CSCI at the ultrastructural level}

Semithin sections of the spinal cords of rats from the control and sham groups showed normal nerve fiber structure (Fig. 4A, B). Following one day after CSCI, the spinal cords at the lesion level showed swollen nerve fibers with apparent reductions in the space between fibers (Fig. 4C). 7 days after CSCI the loss of numerous fibers was evident (Fig. 4D).

At the ultrastructural level the myelinated nerve fibers in spinal cord of the control rats appeared 

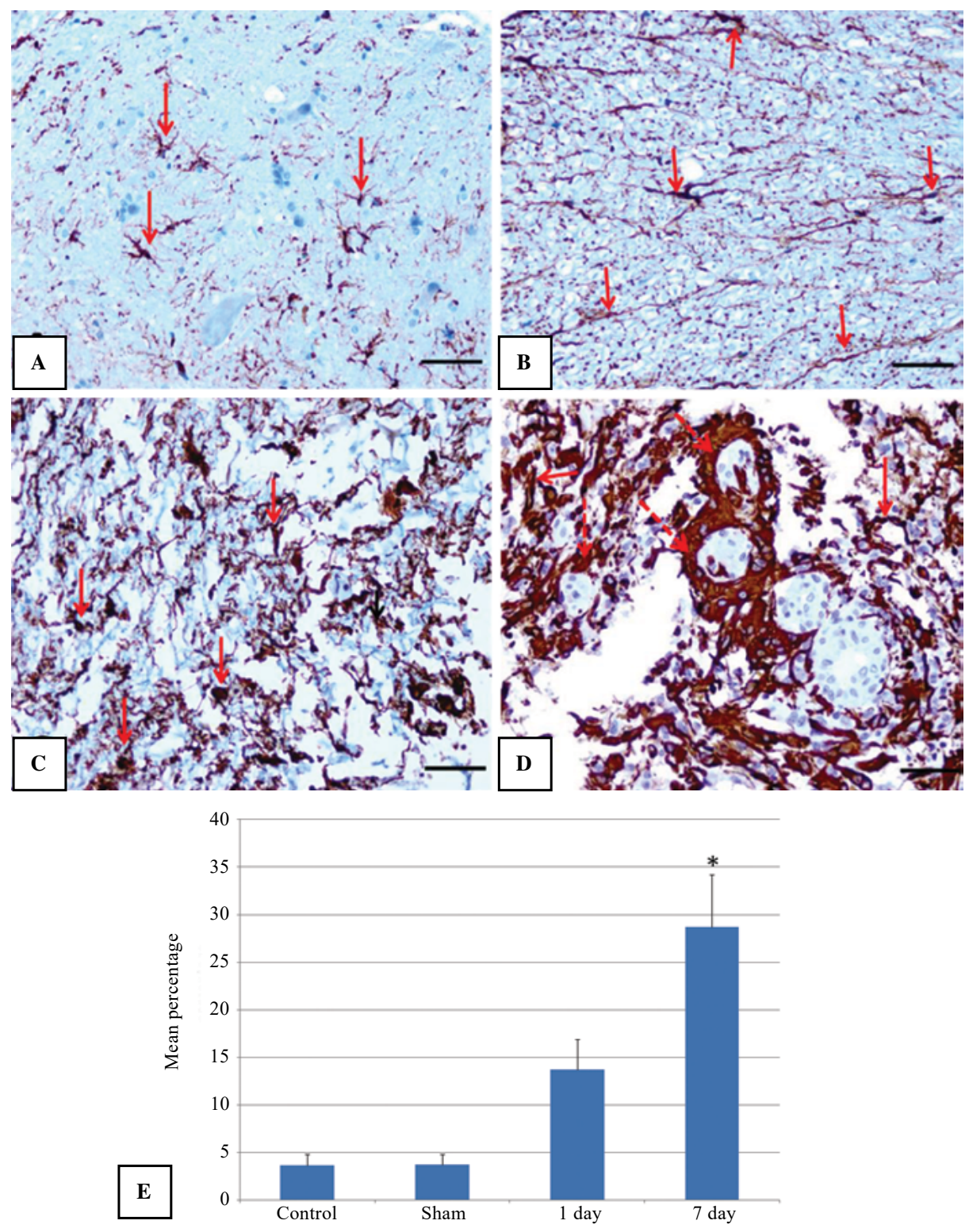

Figure 2. Sections of white matter of the spinal cord of control rats and animals subjected to compressed spinal cord injury (CSCI) as seen by immunohistochemical staining for GFAP. Scattered astrocytes in control (A) and an apparent increase in sham-operated (B) rats. One day after CSCI (C) reactive astrocytes with thick cytoskeletal processes at the lesion level are noted (arrows). Seven days after CSCI (D) localized circumscribed areas of marked reactive astrocytosis (dashed arrows) and abundant reactive astrocytes are noticed. Scale bars A-D, $50 \mu \mathrm{m}(\mathbf{E})$. Bar graph shows a significant increase of the percentage of the mean area of GFAP expression in sections of spinal cord seven days after CSCI. *Significantly different from other groups, $\mathrm{P}<0.05$.

normal; they showed normal axoplasm with well-preserved cellular structures such as normal mitochondria (Fig. 5A). In the sham-operated group, most of the intact myelinated axons revealed normal axoplasm. The myelin sheaths appeared normal, except for some that became swollen (Fig. 5B).

On day 1 after CSCI, the myelinated axons had a swollen appearance with redundant myelin loops that showed apparent thinning. In addition, a loss of cristae was observed in some mitochondria (Fig. $5 \mathrm{C})$. In other sections, areas of myelin splitting had an onion-like appearance. The degeneration of the axoplasm was noted in some axons (Fig. 5D).

Seven days after CSCI, partial and complete areas of demyelination were noticed (Fig. 5E). The layers of the myelin sheath were folded and wrinkled. The myelin lamellae were disorganized and loose (Fig. $5 \mathrm{~F})$.

The measurements of the G-ratio revealed that the proportion of axons diameter to that of axon and myelin sheath was significantly higher in day 1 group as compared with other groups (Fig. 5G). 


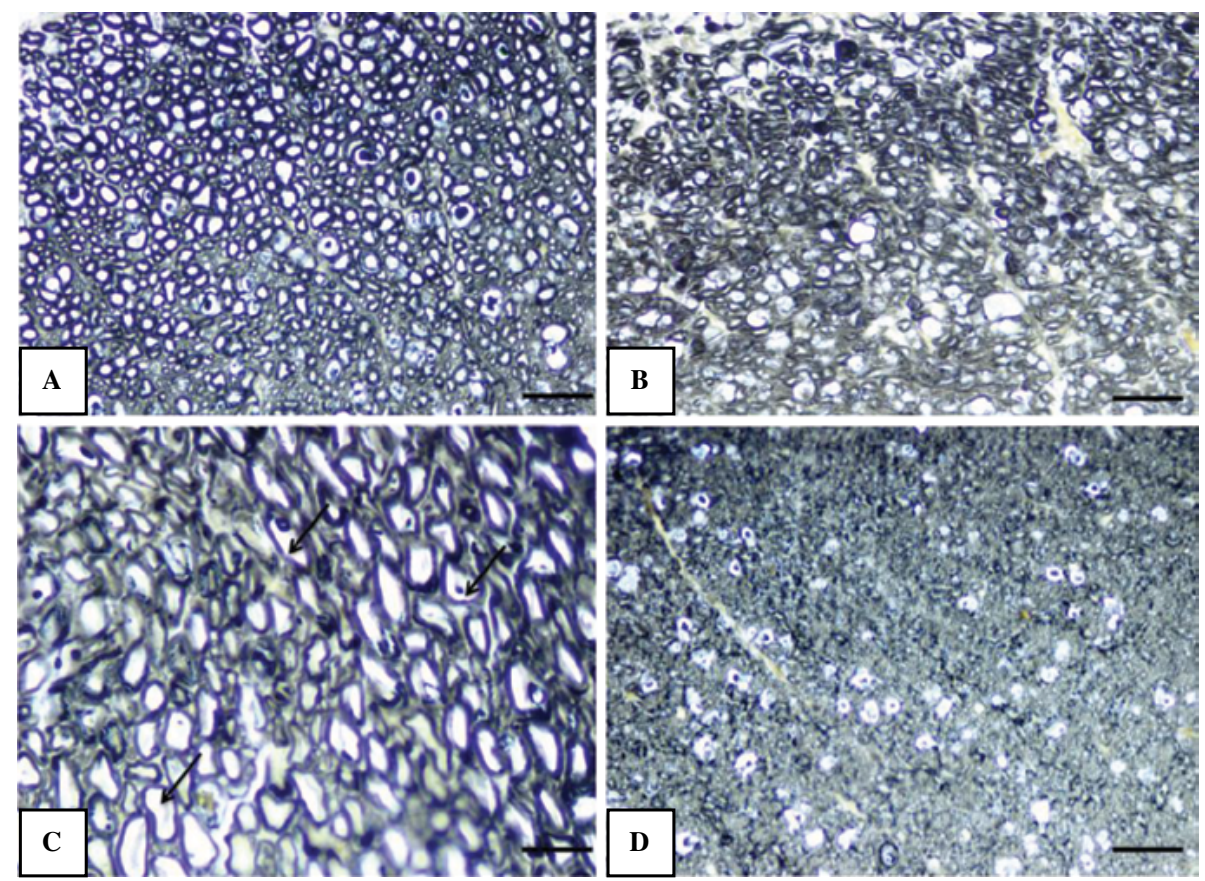

Figure 3. Sections of white matter of the spinal cord of control rats and animals subjected to compressed spinal cord injury (CSCI) as seen by osmic acid staining. Darkly stained intact myelin sheaths reflect normal myelination of nerve fibers in control (A) and sham-operated (B) rats. One day after CSCI (C) nerve fibers became swollen (arrows) and the spaces between fibers were apparently decreased. Seven days after CSCI (D) marked loss of fibers is observed. Scale bars A-D, $50 \mu \mathrm{m}$.
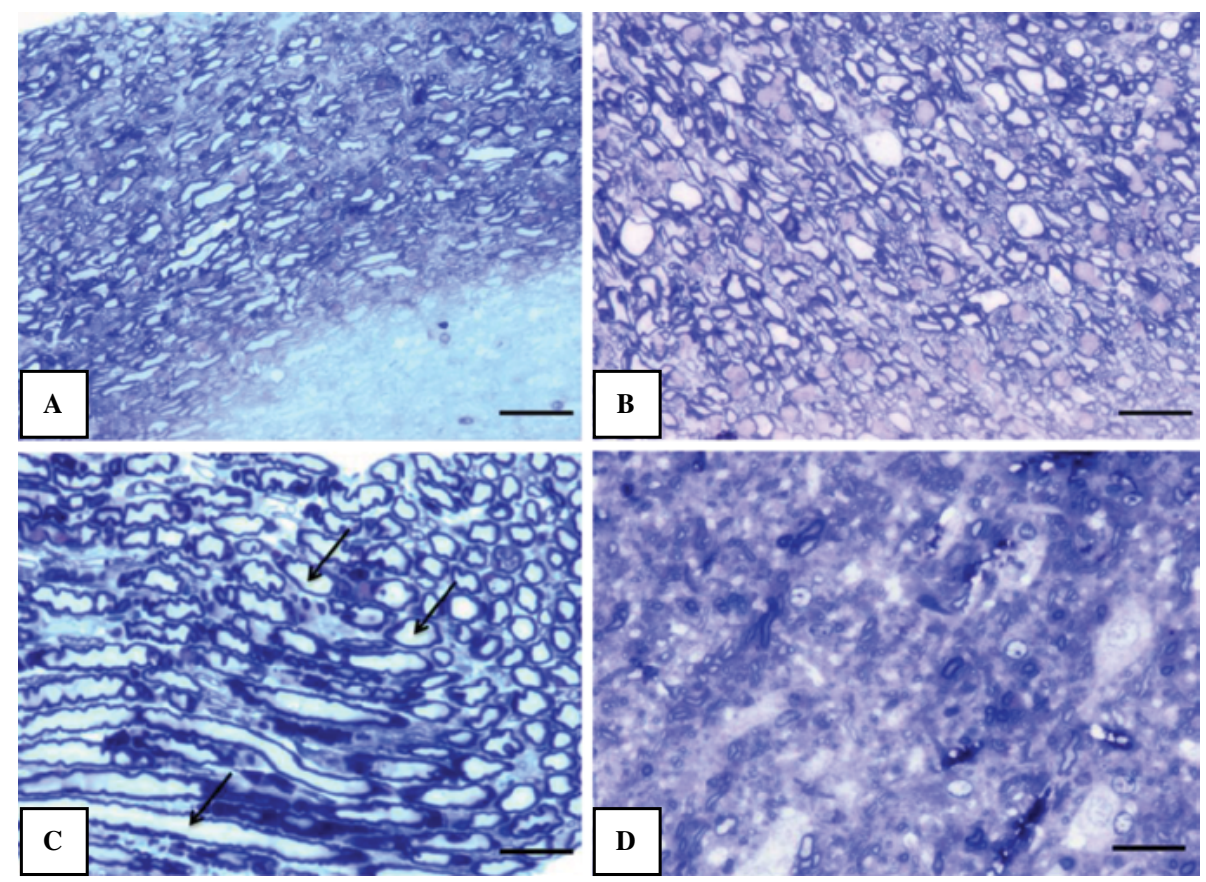

Figure 4. Semithin sections of white matter of the spinal cord of control rats and animals subjected to compressed spinal cord injury (CSCI) as seen by toluidine blue staining. Normal structure of nerve fibers in control (A) and sham-operated (B) rats. One day after CSCI (C) nerve fibers are swollen (arrows) with apparent reduction in the spaces between them. Seven days after CSCI (D) obvious loss of fibers is noted. Scale bars A-D, $50 \mu \mathrm{m}$. 

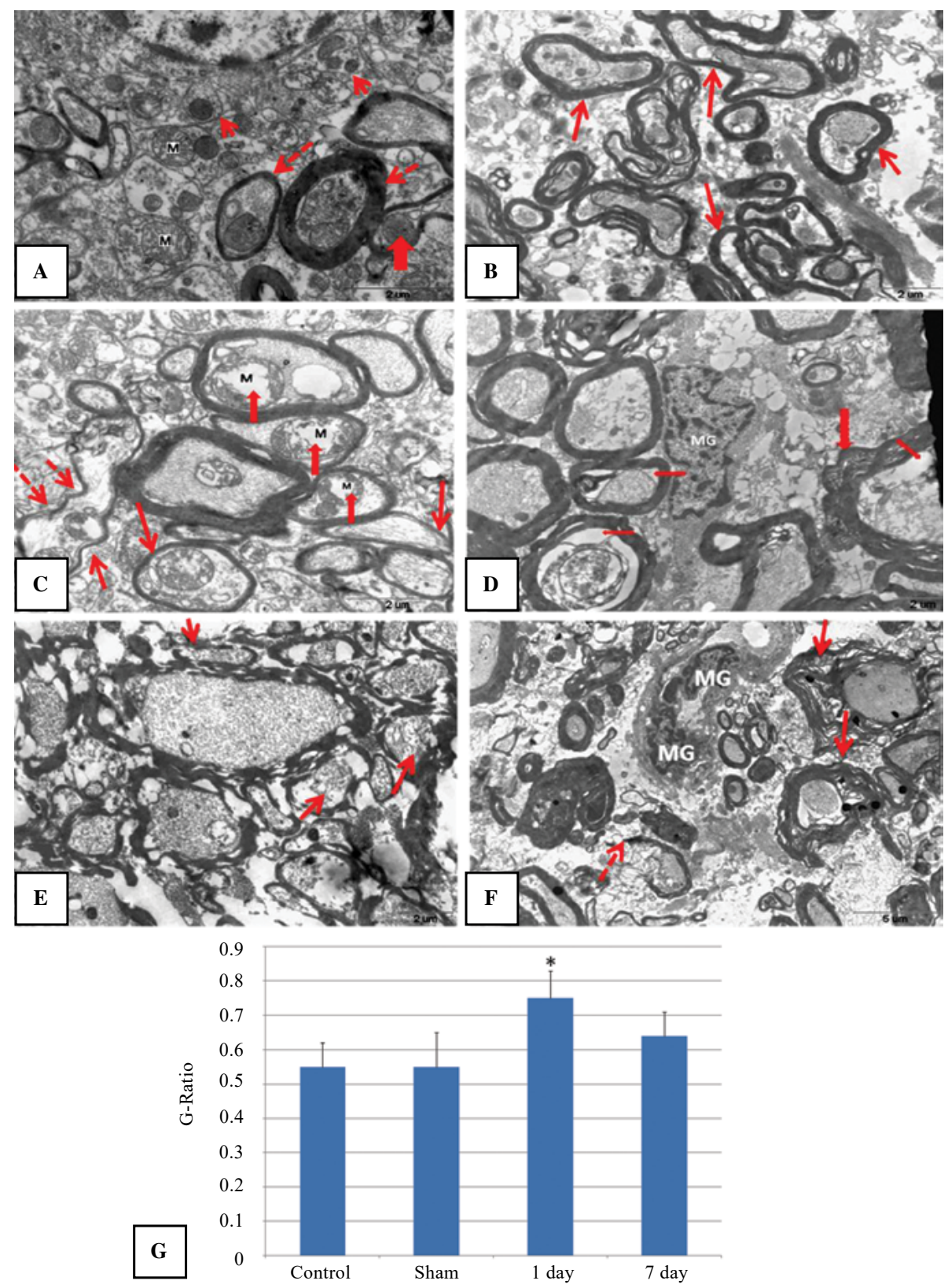

Figure 5. Ultrathin sections of white matter of spinal cord of control rats and animals subjected to compressed spinal cord injury (CSCI) as seen by uranyl acetate and lead citrate staining. Normal structure of myelinated axons with normal axoplasm and well-preserved mitochondria in control (A) and sham-operated (B) rats. C and D: one day after CSCI. In (C) the myelinated axons are swollen (thin arrows) with redundant thin myelin loops (dashed arrows) and loss of cristae in some mitochondria is noticed (thick arrows). In (D) myelin splitting (thick arrow) and degeneration of the axoplasm is noticed. E and F: seven days after CSCI. In (E) areas of demyelination are noticed (arrows). In (F) layers of the myelin sheath are folded and wrinkled and myelin lamellae are disorganized and loose. G. A bar graph shows G-ratio (axon diameter/total fiber diameter) in the studied groups of rats. *Significantly higher value one day after CSCI as compared to other groups, $\mathrm{P}<0.05$. Scale bars: A-E, $2 \mu \mathrm{m} ; \mathrm{F}, 5 \mu \mathrm{m}$.

\section{Discussion}

Compressed spinal cord injuries (CSCIs) are among the most incapacitating lesions that humans can suffer from. A great deal of interest has been aroused in their histopathological, biochemical and functional changes, and particularly in the search for preventive and therapeutic methods [16]. CSCIs impair motor and sensory functions insidiously and progressively, and so they must be considered very serious disorders on the personal and social levels [17]. 
There have been few previous studies of the pathological characteristics of the demyelination induced by CSCI. Suitable models elucidating the morphology and mechanism underlying it in CSCI have seldom been provided. Therefore, in the present study, we chose the clip impact-compression model, which differs from other compression models used in many SCI studies, because after the initial acute impact on the spinal cord due to the rapid closure of the clip blades, there is a period of continuing compression, as the clip remains in its closed position for a predetermined length of time. This temporal effect of the compression injury is not achieved in most other compression models, such as the Infinite Horizons model. The aim of our research was to define the histopathological changes of CSCI with special attention to the ultrastructural study.

In the current study, the T9-10 sections of spinal cord sampled 1 day after CSCI revealed minute hemorrhages, fibrinoid necrosis, numerous punched out areas representing necrosis, and a loss of white matter. Moreover, the spinal cord of rats sampled 7 days after CSCI revealed extended areas of cavitation surrounded by a demarcating rim of numerous astrocytes. Thus, the crushed spinal cord tissue, together with bleeding, was seen after the contusion. It has been assumed that some substances that ooze out from the primary injury site cause the secondary injury characterized by the presence of an ischemic zone that results in tissue destruction and expansion of the secondary injury. This process continues to form liquefied cavities and fibrous trabeculae $[18,19]$. Astrocytes, potential energy source, have a major role in clearing potassium ions and glutamate from the extracellular space and produce numerous cytokines and growth factors [20]. Inflammatory response can lead to axon demyelination and neuroglial apoptosis, with subsequent loss of neurological function [21]. In rats, SCI activates myelin basic protein (MBP)-reactive T cells that are capable of causing neuron inflammation and transient paralysis [22].

In this study, areas of reactive astrocytosis were revealed one day after CSCI, while localized circumscribed areas of reactive astrocytosis and a significant increase in the mean percentage area of GFAP expression were evident after seven days of CSCI. In accordance with these findings, previous studies have found that after SCI astrocytes, the most abundant glial cells in the CNS, transform into reactive astrocytes and undergo dramatic morphological changes [23]. Reactive astrocytes, the major component of glial scars, along with other cells in the spinal cord and blood-borne cells leaking from the damaged blood-spinal cord barrier, participate in the process of scar formation [24]. Generally, scar tissue is classified into two types, fibrotic and glial. Fibrotic scar is primarily composed of invading fibroblasts derived from perivascular and meningeal cells and occupies the epicenter of the lesion area, with sedimentation of the collagen matrix [25].

Glial scars (mainly astrocytic) evolve from the dynamic process of reactive gliosis, which is characterized by complicated and mesh-like processes extended by reactive astrocytes converging on the perilesion zone. It is also postulated that regions of marked tissue degeneration after SCI where astrocytes have become dysfunctional [26] or migrated away [27] in response to the initial injury may be a major factor to the process of secondary degeneration after SCI.

Osmium tetroxide is a contrasting agent of lipids, which are the main components of myelin sheaths. Myelin sheaths can be oxidized and stained by osmium tetroxide, producing a black appearance [28]. Hence, the osmium tetroxide staining applied in this study effectively demonstrated the swelling of nerve fibers one day after CSCI. Meanwhile, seven days after CSCI, the loss of numerous fibers was observed where little or no osmium tetroxide stain was present. Information on demyelination after spinal cord injuries has rarely been reported in terms of neuronal loss, although early pathological studies suggested that demyelination occurs in CSCI pathology [6]. Emerging evidence also suggests that demyelination is an important pathological factor in spinal cord injuries. Given that myelin is important in normal nerve impulse conduction, the loss of the myelin sheaths around axons may significantly contribute to neurological deficits after CSCI [29].

The ultrastructural study in the present work revealed swollen myelinated axons with redundant myelin loops, an apparent thinning of myelin, and myelin splitting with an onion-like appearance one day after CSCI. In addition to these changes, layers of the myelin sheath were folded, wrinkled, and disorganized, with evidence of demyelinated areas seven days after CSCI. Similarly, other authors [30] found that myelin sheaths became swollen, degenerated and were broken down gradually. In addition, the axolemma was separated from the myelin sheath. The axoplasm in the lesion area contained varying degrees of reduction in the mitochondria and microtubules [30].

The documentation and arrangement of oligodendrocyte-remyelinated axons are based on the G-ratio. The G-ratio is the diameter of the axon to the diameter of the axon plus the surrounding myelin. The lower the G-ratio, the bigger is the extent of remyelination [31]. Conversely, a higher G-ratio indicates thinner myelin sheaths and incomplete remyelination [32]. 
A high occurrence of very thin myelin surrounding lesion zones was observed even years after spinal cord injury. The regenerated myelin sheaths have been classified in pathological conditions as falling into a very high G-ratio range of 0.9 to 1.0 [33]. These findings were in agreement with our observation where the G-ratio had significantly increased one day after the CSCI as a result of the swelling of axon and hematoma immediately after the trauma. On the other hand, at seven days after CSCI, the G-ratio was smaller than that at day 1 , but was still higher than its value in the control and sham-operated animals, which indicates thinner myelin sheaths and incomplete remyelination.

\section{Conclusions}

The study revealed that rat thoracic cord clip compression model is a clinically relevant spinal cord injury model leading to progressive histopathological changes and demyelination which were intensified with the duration of time after spinal cord injury. Thus, in human CSCI prompt medical intervention is vital for the prevention of further disabilities.

\section{Acknowledgements}

This project was funded by the Deanship of Scientific Research (DSR), King Abdulaziz University, Jeddah, under grant No. 10/248/1433. The authors thank DSR for financial and technical support.

\section{Conflict of interest}

The authors report no conflict of interest.

\section{References}

1. MFA. Spinal cord injuries in the developing world. In: International encyclopedia of rehabilitation. Buffalo University, New York; 2013.

2. Fehlings MG, Tator $\mathrm{CH}$. A review of experimental models of acute spinal cord injury. In: Spinal cord dysfunction: assessment. Oxford University, Oxford; 1988.

3. Alhoseini MS, Movaghar VR. Orthopedics, physical medicine and rehabilitation animal models in traumatic spinal cord injury. In: Dionyssiotis Y. ed. Topics in paraplegia. InTech, Rijeka, Croatia; 2014.

4. Lerman CT, Altevogt BM, Joy JE, Johnson RT. Progression of spinal cord injury. In: Spinal cord injury: progress, promise and priorities. The National Academies Press, Washington DC; 2005.

5. Consortium for Spinal Cord Medicine. Early acute management in adults with spinal cord injury: a clinical practice guideline for health-care professionals. J Spinal Cord Med. 2008; 31(4): 403-479, indexed in Pubmed: 18959359.

6. Faulkner JR, Herrmann JE, Woo MJ, et al. Reactive astrocytes protect tissue and preserve function after spinal cord in- jury. J Neurosci. 2004; 24(9): 2143-2155, doi: 10.1523/JNEUROSCI.3547-03.2004, indexed in Pubmed: 14999065.

7. McGavern DB, Murray PD, Rodriguez M. Quantitation of spinal cord demyelination, remyelination, atrophy, and axonal loss in a model of progressive neurologic injury. J Neurosci Res. 1999; 58(4): 492-504, indexed in Pubmed: 10533042.

8. McGavern DB, Zoecklein L, Drescher KM, et al. Quantitative assessment of neurologic deficits in a chronic progressive murine model of CNS demyelination. Exp Neurol. 1999; 158(1): 171-181, doi: 10.1006/exnr.1999.7082, indexed in Pubmed: 10448429.

9. Bakar B, Kose E, Ayva SK, et al. Effects of low-dose methotrexate in spinal cord injury in rats. Turk J Trauma Emergency Surg. 2013; 19(4): 285-293, doi: 10.5505/tjtes.2013.65475.

10. Bancroft JD, Gamble M. Hematoxylin and eosin, connective tissue and stain, carbohydrates. In: Theory and practice in histological techniques. 6th ed. Churchill-Livingstone, Edinburgh; 2008: 121-186.

11. Afsari ZH, Renno WM, Abd-El-Basset E. Alteration of glial fibrillary acidic proteins immunoreactivity in astrocytes of the spinal cord diabetic rats. Anat Rec (Hoboken). 2008; 291(4): 390-399, doi: 10.1002/ar.20678, indexed in Pubmed: 18360886.

12. Hashish HA. Alteration of glial fibrillary acidic protein immunoreactivity in astrocytes of the cerebellum of diabetic rats and potential effect of insulin and ginger. Anat Physiol. 2015; 5(1), doi: 10.4172/2161-0940.1000167.

13. Wei Lp, He Fc, Chen Xw, et al. Osmic acid staining of myelin sheath in normal and regenerated peripheral nerves. Chin J Traumatol. 2007; 10(2): 86-89, indexed in Pubmed: 17371618.

14. Bozzola JJ, Russel LD. Electron microscopy: principles and techniques for biologists. 2nd ed. Jones and Bartlet Publishers, Sudbury; 1998.

15. Chomiak T, Hu B. What is the optimal value of the G-ratio for myelinated fibers in the rat CNS? A theoretical approach. PLoS One. 2009; 4(11): e7754, doi: 10.1371/journal. pone.0007754, indexed in Pubmed: 19915661.

16. Lebl DR, Hughes A, Cammisa FP, et al. Cervical spondylotic myelopathy: pathophysiology, clinical presentation, and treatment. HSS J. 2011; 7(2): 170-178, doi: 10.1007/s11420011-9208-1, indexed in Pubmed: 22754419.

17. Tator $\mathrm{CH}$, Fehlings MG. Review of the secondary injury theory of acute spinal cord trauma with emphasis on vascular mechanisms. J Neurosurg. 1991; 75(1): 15-26, doi: 10.3171/jns.1991.75.1.0015, indexed in Pubmed: 2045903.

18. Beattie MS, Bresnahan JC. Cell death, repair, and recovery of function after spinal cord contusion injuries in rats. In: Neurobiology of spinal cord injury. Humana Press, New Jersey; 2000.

19. Yu WRu, Fehlings MG. Fas/FasL-mediated apoptosis and inflammation are key features of acute human spinal cord injury: implications for translational, clinical application. Acta Neuropathol. 2011; 122(6): 747-761, doi: 10.1007/s00401-0110882-3, indexed in Pubmed: 22038545.

20. Kettenmann H, Ransom B. The concept of neuroglia: a historical prespective. In: Neuroglia. Oxford University Press, New York; 2005.

21. Liu H, Shubayev VI. Matrix metalloproteinase-9 controls proliferation of $\mathrm{NG} 2+$ progenitor cells immediately after spinal cord injury. Exp Neurol. 2011; 231(2): 236-246, doi: 10.1016/j. expneurol.2011.06.015, indexed in Pubmed: 21756907.

22. Tian Ds, Xie Mj, Yu Zy, et al. Cell cycle inhibition attenuates microglia induced inflammatory response and alleviates neuronal cell death after spinal cord injury in rats. Brain Res. 2007; 1135(1): 177-185, doi: 10.1016/j.brainres.2006.11.085, indexed in Pubmed: 17188663. 
23. Wilhelmsson U, Bushong EA, Price DL, et al. Redefining the concept of reactive astrocytes as cells that remain within their unique domains upon reaction to injury. Proc Natl Acad Sci U S A. 2006; 103(46): 17513-17518, doi: 10.1073/pnas.0602841103, indexed in Pubmed: 17090684.

24. Fawcett JW, Asher RA. The glial scar and central nervous system repair. Brain Res Bull. 1999; 49(6): 377-391, indexed in Pubmed: 10483914.

25. Göritz C, Dias DO, Tomilin N, et al. A pericyte origin of spinal cord scar tissue. Science. 2011; 333(6039): 238-242, doi: 10.1126/science.1203165, indexed in Pubmed: 21737741.

26. Fitch MT, Doller C, Combs CK, et al. Cellular and molecular mechanisms of glial scarring and progressive cavitation: in vivo and in vitro analysis of inflammation-induced secondary injury after CNS trauma. J Neurosci. 1999; 19(19): 8182-8198, indexed in Pubmed: 10493720.

27. Zhao X, Ahram A, Berman RF, et al. Early loss of astrocytes after experimental traumatic brain injury. Glia. 2003; 44(2): 140-152, doi: 10.1002/glia.10283, indexed in Pubmed: 14515330.

28. Wei Lp, He Fc, Chen Xw, et al. Osmic acid staining of myelin sheath in normal and regenerated peripheral nerves. Chin J Traumatol. 2007; 10(2): 86-89, indexed in Pubmed: 17371618.
29. Vaquero J, Zurita M, Oya S, et al. Early administration of methylprednisolone decreases apoptotic cell death after spinal cord injury. Histol Histopathol. 2006; 21(10): 1091-1102, doi: 10.14670/HH-21.1091, indexed in Pubmed: 16835831.

30. Huang SQ, Tang CL, Sun SQ, et al. Demyelination initiated by oligodendrocyte apoptosis through enhancing endoplasmic reticulum-mitochondria interactions and Id 2 expression after compressed spinal cord injury in rats. CNS Neurosci Ther. 2014; 20(1): 20-31, doi: 10.1111/cns.12155, indexed in Pubmed: 23937638.

31. Stikov N, Campbell JSW, Stroh T, et al. In vivo histology of the myelin G-ratio with magnetic resonance imaging. Neuroimage. 2015; 118: 397-405, doi: 10.1016/j.neuroimage.2015.05.023, indexed in Pubmed: 26004502.

32. Gu Z, Li F, Zhang YiP, et al. Apolipoprotein E mimetic promotes functional and histological recovery in lysolecithin-induced spinal cord demyelination in mice. J Neurol Neurophysiol. 2013; 2014(Suppl 12): 10, doi: 10.4172/21559562.S12-010, indexed in Pubmed: 25642353.

33. Totoiu MO, Keirstead HS. Spinal cord injury is accompanied by chronic progressive demyelination. J Comp Neurol. 2005; 486(4): 373-383, doi: 10.1002/cne.20517, indexed in Pubmed: 15846782.

Submitted: 23 April, 2016

Accepted after reviews: 19 March, 2017 Available as AoP: 23 March, 2017 